\title{
Typologie Et Déterminants Des Stratégies D'adaptation Aux Changements Climatiques En Riziculture Au Nord Et Centre Du Bénin
}

\author{
Lionel Ulrich Ayedegue, \\ Ingénieur Agronome, Doctorant en Economie des Ressources Naturelles, \\ Université de Parakou, Faculté d'Agronomie, \\ Laboratoire d'Analyse et de Recherche sur les Dynamiques Economique et \\ Sociale, Parakou, Bénin \\ Kassim Issaka, Docteur en Agroéconomie \\ Jacob Afouda Yabi, Professeur Titulaire en Agroéconomie \\ Université de Parakou, Faculté d'Agronomie, \\ Laboratoire d'Analyse et de Recherche sur les Dynamiques Economique et \\ Sociale, Parakou, Bénin
}

Doi:10.19044/esj.2020.v16n6p206 ～URL:http://dx.doi.org/10.19044/esj.2020.v16n6p206

\section{Resume}

La combinaison des stratégies d'adaptation semble être aujourd'hui les meilleures options qui permettent d'atténuer les effets des changements climatiques. C'est dans ce cadre que cette étude vise à réaliser une typologie des stratégies d'adaptation développées en riziculture au Bénin ainsi que les déterminants. Sur la base de la théorie des dynamiques adaptatives de Chia et al., l'hypothèse de travail est que les producteurs combinent plusieurs stratégies en fonction de leur situation pour faire face aux changements climatiques. L'étude s'est basée sur un échantillon de 144 riziculteurs enquêtés à l'aide d'un questionnaire et des entretiens. L'Analyse Factorielle des Correspondances Multiples (AFCM) suivie d'une Classification Hiérarchique Ascendante (CHA) ont permis de réaliser la typologie. Le modèle logit multinomial a permis d'identifier les déterminants de l'adoption des stratégies catégorisées. De l'AFCM, trois (03) catégories de stratégies se dégagent et se diffèrent par le contact des producteurs avec un projet sur le changement climatique, le niveau d'instruction, la zone agroécologique, le sexe et les informations sur les prévisions climatiques. Les riziculteurs de la Classe 1 adoptent et combinent les stratégies telles que les rotations culturales, l'accroissement des superficies cultivées, une plus grande utilisation d'engrais chimique et l'utilisation des variétés résistantes ; ceux de la classe 2 combinent le semis direct et l'utilisation des variétés de cycle court alors que ceux de la Classe 3 s'adaptent en combinant la diversification culturale et l'introduction 
de nouvelles spéculations. Les résultats du modèle logit multinomial révèlent que le sexe, le contact avec un projet sur le changement climatique, l'utilisation de la fumure organique et l'accès à l'information sur les prévisions climatiques sont les facteurs qui déterminent les stratégies. Les agents du développement de la filière riz devraient orienter des actions de soutien en tenant compte de ces résultats pour accompagner les acteurs.

Mots clés : Caractérisation, Changement Climatique, Bénin, Riz, Stratégie D'adaptation

\title{
Typology and Determinants of Climate Change Adaptation Strategies in Rice Growing in North Benin
}

\author{
Lionel Ulrich Ayedegue, \\ Ingénieur Agronome, Doctorant en Economie des Ressources Naturelles, \\ Université de Parakou, Faculté d'Agronomie, \\ Laboratoire d'Analyse et de Recherche sur les Dynamiques Economique et \\ Sociale, Parakou, Bénin \\ Kassim Issaka, Docteur en Agroéconomie \\ Jacob Afouda Yabi, Professeur Titulaire en Agroéconomie \\ Université de Parakou, Faculté d'Agronomie, \\ Laboratoire d'Analyse et de Recherche sur les Dynamiques Economique et \\ Sociale, Parakou, Bénin
}

\begin{abstract}
Combining adaptation strategies are now options that can mitigate the effects of climate change. It is in this context that this study aims to make a typology of the adaptation strategies developed in rice growing in Benin. Based on the theory of adaptive dynamics of Chia et al., The working hypothesis is that producers combine several strategies depending on their situation to cope with climate change. The study was based on a sample of 144 rice farmers surveyed using a questionnaire and interviews. The Factorial Analysis of Multiple Correspondences (AFCM) followed by an Ascending Hierarchical Classification (CHA) made it possible to carry out the typology. The multinomial Logit model identified the determinants of the adoption of categorized strategies. From AFCM, three (03) categories of strategies are obtained and differ by the contact of producers with a project on climate change, level of education, agro-ecological zone, gender and information on
\end{abstract}


forecasts climate. Class 1 rice farmers combine strategies such as crop rotations, increasing the area cultivated, Greater use of chemical fertilizers and the use of resistant varieties while those of Class 2 combine direct seeding and use of short cycle varieties and those of Class 3 combine crop diversification and the introduction of new crop. The multinomial logit model reveals gender, contact with a climate change project, the use of organic manure, and access to information on climate forecasts. These different determinants are the factors on which development agents in the rice sector should direct support actions to support actors.

Keywords: Characterization, Climate Change, Benin, Rice, Adaptation Strategy

\section{Introduction}

La plupart des pays d'Afrique de l'Ouest sont touchés par de nombreux effets néfastes du changement climatique. Au cours de ces dernières années, on observe une augmentation significative de leur fréquence et de leur intensité attribuable au réchauffement du climat (Gemenne et al., 2017). Ce réchauffement a une grande influence sur les productions agricoles et les revenus des ménages ruraux africains (Oumarou et al., 2017). Au Bénin, ces influences se manifestent par les inondations, les sécheresses, la modification du calendrier agricole, les vagues de chaleur et la faible productivité sont autant des effets les plus tangibles qui affectent les ménages agricoles (Reeves et al., 2016). A cela, s'ajoutent la dégradation des sols, l'érosion côtière, la déforestation, la faible diversification culturale entrainant la dégradation des bas-fonds et bassins qui menacent la sécurité alimentaire et amplifient la pauvreté (Breumier et al., 2018). Les céréales, en particulier le riz pluvial, sont les plus touchées par les variabilités et le changement climatique au Bénin. En effet, le riz occupe la troisième place dans les systèmes de production et dans l'alimentation la deuxième céréale la plus consommée (2/3 ménages) par les populations après le maïs (Macauley \& Ramadjita, 2015). Il représente environ $80 \%$ des productions céréalières totales des zones de production au Bénin (Houssou et al., 2016). Ainsi, des actions telles que l'aménagement, la valorisation des bas-fonds et des bassins ainsi que l'irrigation des périmètres sont des actions menées par les acteurs du développement pour faciliter la production du riz (Atidegla et al., 2017). Mais le véritable problème de la culture du riz est l'influence du climat car le riz est une culture exigeante en eau (Assouma et al., 2019a). De plus, les ressources en eau connaissent une diminution progressive en témoignent plusieurs travaux réalisés au Bénin sur la vulnérabilité des ressources en eau (Totin, 2005 ; Dakossi, 1994) combiné à l'inefficacité de son utilisation sur les périmètres rizicoles. En effet, des économies importantes d'utilisation de l'eau irriguée de l'ordre de $72 \%$ 
peuvent être réalisées en améliorant la façon dont l'eau est allouée en riziculture (Ayedegue et al., 2018).

Dans ce contexte et pour assurer l'autosuffisance alimentaire et la stabilité des revenus des riziculteurs, les stratégies d'adaptation sont aujourd'hui les seules alternatives pour réduire les effets des variabilités climatiques. En effet, l'adaptation au changement climatique renvoie à « l'ajustement dans les systèmes naturels ou humains en réponse à des changements climatiques actuels ou attendus, ou à leurs effets, qui atténue les dommages ou en valorise les bénéfices » (Organisation des Nations Unies pour l'Alimentation et l'Agriculture [FAO], 2007). Ainsi, les adaptations sont catégorisées selon plusieurs critères comportant, l'horizon (court ou long terme), le temps (réactive ou anticipée), la forme (technique, institutionnel, légal, comportemental ou éducationnel) et les acteurs impliqués (Fussel, 2007 ; Smit et Skinner, 2002).

Les riziculteurs perçoivent les effets du changement climatique et développent plusieurs stratégies d'adaptation (Abdou et al., 2014) de manière endogène pour réduire les effets néfastes de ces variabilités climatiques sur la production. De plus, des stratégies d'adaptation ont été développées par les centres d'innovations et institutions de recherche agricoles et mises en œuvre par les producteurs des pays en voie de développement et sous-développés (Kabir et al., 2017 ; Alauddin et Sarker, 2014 ; Zorom et al., 2013 ; Deressa et al., 2009 ; Ouédraogo et al., 2010). Les stratégies développées par les riziculteurs sont pour la plupart anticipatives ou réactives et on distingue l'utilisation de variétés améliorées, l'utilisation des variétés résistantes, l'irrigation de complément, la migration des zones à risques, l'utilisation des intrants adaptés (Naswem et al., 2016). Par ailleurs, des travaux sont effectués de façon globale sur les typologies des perceptions du changement climatique (Gnanglè et al., 2012 ; Arodokoun et al., 2012) et les typologies des stratégies d'adaptation aux changements climatiques en agriculture (Idrissou et al., 2017 ; De Munck, 2013 ; Ouédraogo et al., 2010). Mais la recherche sur la caractérisation des stratégies d'adaptation surtout en lien avec la riziculture reste encore très peu connue. De plus, plusieurs études se sont concentrées sur le niveau mondial, régional ou national (Challinor et al., 2014). Ces études ont joué un rôle crucial dans la planification des stratégies d'adaptation au niveau macroéconomique. Cependant, les études portant sur les communautés, les ménages et les exploitations agricoles sont nécessaires pour identifier et concevoir des stratégies d'adaptation efficaces au niveau local. Aussi, les agriculteurs pratiquent des stratégies adaptation différents selon le pays et fonction des facteurs climatiques, sociaux, économiques et institutionnels (Below et al., 2012 ; Deressa et al., 2009). Alors, la présente étude essaie de combler cette lacune dans le monde de la recherche scientifique et se propose de faire la Typologie des stratégies d'adaptation réelles adoptées par les 
riziculteurs des bassins de production du riz au Bénin en identifiant les déterminants de l'adoption de ces stratégies.

\section{Materiel Et Methodes}

\section{Zone D'etude}

L'étude a été réalisée dans le centre et le Nord du Bénin. Le choix de ces zones part du fait que ses conditions agro-écologiques sont très favorables à la culture du riz (Assouma et al., 2019a) et regroupent 3 des 4 bassins rizicoles du Bénin. Le centre du Bénin et le Nord-Bénin sont des zones qui regorgent plus des bas-fonds et des bassins où la production du riz se fait à une échelle très importante. Certaines communes de ces zones ont de très grandes potentialités en matière de production, de transformation et de la commercialisation du riz. Ainsi, ceci a suscité l'implantation de deux (02) usines d'étuvage moderne GEM (qui est un système d'étuvage du riz amélioré) dans les zones (une au centre et une autre Sud) notamment à Glazoué et Malanville. Les communes de Malanville, Glazoué et Matéri ont été retenues pour cette étude. Trois villages ont été choisis par commune pour réaliser les enquêtes socio-économiques. Les villages concernés ont été : Magoumi, Agouagon et Zaffé dans la commune de Glazoué, Madécali, Kotchi, et Toumboutou dans la commune de Malanville et, Kakini-Séri, Holli et Dassari dans la commune de Matéri (figure1). Aussi bien les communes et les villages ont été choisis sur la base des critères suivants : poids en matière de la production du riz ; zones vulnérables aux manifestations du changement climatique et récurrence à l'insécurité alimentaire. La production du riz représente pour ces villages une activité non seulement socioéconomique mais aussi une garantie de la sécurité alimentaire. Les résultats du Programme National d'Adaptation aux changements climatiques (PANA-Bénin, 2008) ont permis de choisir les zones les plus vulnérables aux manifestations du changement climatique. 


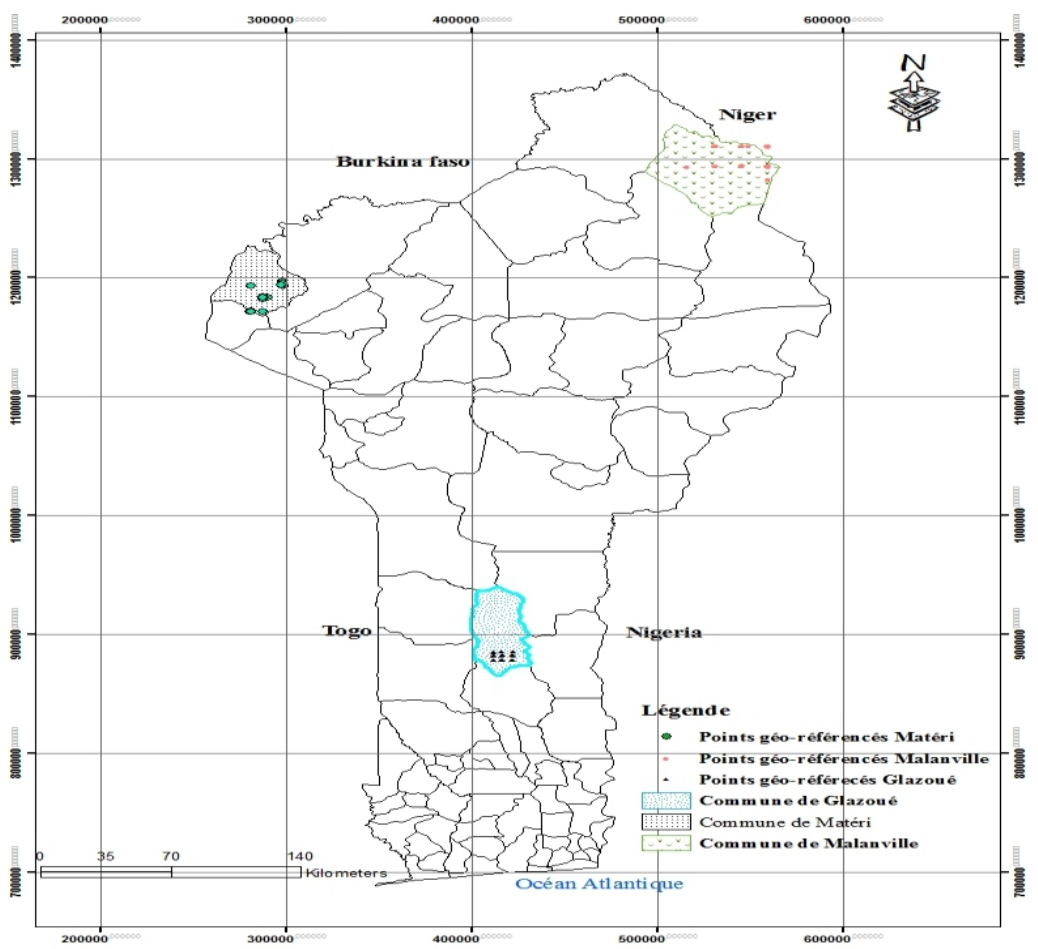

Figure 1. Carte de la zone d'étude

Source : Ayedegue et al. (2018)

\section{Methodes}

\section{Cadre Théorique}

\section{Théorie Du Choix Rationnel}

Plusieurs stratégies d'adaptation sont développées sous différentes formes et échelles de mise en ouvre pour faire face aux changements climatiques en milieu urbain comme en milieu rural (De Munck, 2013). Certaines stratégies sont développées par les producteurs et d'autres par la recherche scientifique. Le riziculteur a une multitude de stratégies d'adaptation parmi lesquelles il opère son choix en fonction de sa capacité (capital humain et financier, terres cultivables; etc.) et de la zone agro écologique à laquelle il appartient. Le riziculteur est alors supposé être rationnel avec un comportement de maximisation sous contrainte définissant sa position d'équilibre.

Selon Boudon (2004), le choix rationnel des stratégies d'adaptation par le riziculteur repose sur trois principes (l'individualisme, la compréhension et la rationalité). Le riziculteur choisit ainsi une ou des stratégies selon ses actions, croyances ou son attitude individuelle face aux stratégies choisies. Tous ceux-ci, suite à la compréhension du riziculteur des stratégies d'adaptation choisies. Ensuite, le riziculteur adhère à une croyance ou entreprend une action parce qu'elle fait sens pour lui, en d'autres termes, que 
la cause principale des actions, croyances, etc., du sujet réside dans le sens qu'il leur donne, plus précisément dans les raisons qu'il a de les adopter ( $l a$ rationalité). Ces raisons sont plus basées sur la «mentalité primitive », la « pensée sauvage » ou la «violence symbolique », ces notions faisant appel à des mécanismes opérant à l'insu du sujet, à l'instar des processus chimiques dont il est le siège. Il n'implique pas cependant que le sujet soit clairement conscient du sens de ses actions et de ses croyances du fait qu'il a une panoplie de stratégies parmi lesquelles il opère son choix et aussi de la gravité des impacts du changement climatique dans son exploitation.

\section{Théorie du comportement adaptatif du producteur en agriculture familiale}

La théorie du comportement adaptatif constitue un dépassement des principales limites de la théorie néoclassique de la production, à savoir le caractère exogène de la fonction de production et de la fonction d'objectif (Chia et al., 2014). Ainsi, Chia et al., (2014) ont montré que cette théorie fait affiliation avec la théorie économique de production et sert de base à la théorie de gestion des exploitations agricoles. Plus loin, ces auteurs évoquent que le point de départ de la théorie du comportement adaptatif du producteur est la reconnaissance d'une technologie pour être mise en œuvre, en toute décision d'action (par exemple le choix d'une stratégie d'adaptation au changement climatique) requiert une série d'actions plus élémentaires (choisir la parcelle, choisir la variété, etc.) requérant chacune une ou plusieurs décisions. À chaque décision correspond la poursuite d'un objectif.

L'action vise alors selon Chia et al., (2014) à adapter la situation aux objectifs. Mais l'adaptation est à double sens :

- le riziculteur doit aussi choisir ses objectifs, et donc les adapter en fonction de sa situation, sous peine autrement de choisir des objectifs utopiques. Les premiers sont parfois vagues et implicites ; ils sont réexaminés à la lumière des difficultés rencontrées par les acteurs dans la mise en œuvre de leurs décisions.

- la situation, elle n'est jamais parfaitement connue dans tous les détails. C'est en agissant que le riziculteur prend mieux conscience des contraintes de sa situation. Il modifie alors sa perception de sa propre situation.

Ces théories attribuent aux agents un comportement rationnel qui en raison d'un certain nombre de préférences dénotent une recherche du plus grand profit pour le moindre mal.

\section{Cadre empirique}

La littérature offre une gamme exhaustive de stratégies d'adaptation des riziculteurs au changement climatique parmi lesquelles on a : l'utilisation 
de variétés améliorées (Naswem et al. 2016; Arimi, 2014), la culture de variétés résistantes à la sécheresse (Khanal et al., 2018 ; Onyegbula et Oladeji, 2017 ; Idoma, et al. 2017 ; Roco et al. 2017 ; Naswem et al. 2016), la culture de variétés résistantes à l'inondation (Khanal et al. 2018 ; Onyegbula et Oladeji, 2017). Khanal et al. (2018) ; Onyegbula et Oladeji (2017) ont souligné le rôle important de l'utilisation des variétés résistantes aux parasites et aux maladies comme une stratégie d'adaptation des riziculteurs au changement climatique tandis que les travaux menés par Khanal et al. (2018) ; Shrestha et al. (2018) ; Onyegbula et Oladeji (2017) ; Roco et al. (2017) ; Idoma et al. (2017) ; Arimi (2014) ; Sabugo (2013) ont conclu que la modification de la date de semis est la principale stratégie d'adaptation des riziculteurs au changement climatique. Naswem et al. (2016) en travaillant sur le riz dans la municipalité du Katsina-Ala au Nigéria ont eu comme résultats de stratégies d'adaptation des riziculteurs au changement climatique; la migration de la zone de risque climatique ; l'utilisation modérée de produits agrochimiques et d'engrais ; le changement de l'utilisation des produits chimiques, des engrais et des pesticides ; la culture multiple; l'adoption des stratégies recommandées de production de riz amélioré; le recyclage des déchets; l'amélioration des compétences de gestion des agriculteurs. Onyegbula et Oladeji (2017) en effectuant les mêmes travaux dans les Etats d'Ebonyi, d'Ekiti et du Niger au Nigéria ont trouvé comme stratégies d'adaptation des riziculteurs le désherbage continu et fréquent; l'utilisation de la lutte antiparasitaire intégrée; l'utilisation appropriée d'engrais; le semis du riz avec des précipitations précoces; la récolte précoce des cultures; l'utilisation d'informations météorologiques opportunes et adéquates; l'utilisation des charmes pour effrayer les oiseaux; le drainage de l'excès d'eau dans le champ pour éviter les engorgements d'eau; la formation d'un groupe d'agriculteurs pour financer l'adaptation; les services de vulgarisation améliorés utilisant des outils TIC modernes; l'assistance aux écoles de terrain pour améliorer les méthodes de culture. Arimi (2014) identifie les déterminants des stratégies appropriées d'adaptation au changement climatique utilisées par les riziculteurs du sud-ouest du Nigeria, dans l'espoir d'atténuer les impacts négatifs du changement climatique sur la production de riz. Il arrive à la conclusion que la recherche des informations d'alerte constitue une stratégie d'adaptation utilisée par les riziculteurs de la zone d'étude. En étudiant l'effet des stratégies d'adaptation au changement climatique des riziculteurs sur la production du riz dans la région d'Agatu au Nigéria, Idoma et al. (2017) ont eu comme principales stratégies d'adaptation utilisées par les riziculteurs la diversification d'activités non agricoles, le paillage de rizières. Khanal et al. (2018) ont exploré au Népal, les facteurs qui influencent la prise de décision des agriculteurs lors de l'adoption de stratégies d'adaptation au changement climatique et l'impact de ces adaptations sur les 
rendements agricoles. L'analyse des résultats révèlent que les riziculteurs pratiquent les stratégies d'adaptation : utilisation des variété à cycle court, la modification du lieu de plantation des variétés, l'augmentation de la quantité de semence, le semis direct, la levée de la dormance, l'amélioration et l'augmentation de l'irrigation, la construction de cours d'eau lors de fortes pluies, la réduction du travail du sol, l'augmentation du désherbage, les techniques de conservation des sols, l'amélioration et l'augmentation de l'utilisation d'engrais chimiques, l'amélioration et l'augmentation de l'utilisation de fumier, l'utilisation davantage de pesticides. Roco et al. (2017) ont analysé l'impact de l'adaptation au changement climatique sur la productivité des cultures annuelles dans les municipalités de Pencahue, Cauquenes, San Clemente, Parral au Chili. Les résultats révèlent l'utilisation de goutte à goutte et arroseurs, le boisement, le zéro labour, l'utilisation de systèmes d'accumulation d'eau, l'utilisation d'engrais vert, l'utilisation de paillage, l'utilisation de cultures de couverture, l'utilisation de tuyaux et de pompes pour l'irrigation, la mise en place de tranchées d'infiltration, le nettoyage de canaux. Shrestha et al. (2018) ont analysé les stratégies d'adaptation au changement climatique dans l'agriculture dans quatre villages du Myanmar et du Cambodge. Ils concluent que des variétés de cultures, des équipements de culture et des modifications de la superficie cultivée sont les stratégies utilisées par les agriculteurs de la zone d'étude. Yabi et al. (2017) ont mené une étude dans la commune de Malanville au Bénin afin d'identifier les déterminants du niveau de production du riz en saison pluvieuse et en contre saison. Pour leur étude, ils ont utilisé la gestion des inputs comme une stratégie adaptation aux variations climatiques inter-saisonnières.

\section{Echantillonnage}

Les unités d'observation sont les producteurs du riz. Dans chaque village sélectionné, 16 riziculteurs ont été constitués de manière aléatoire à choix raisonné (au moyen de la table des nombres aléatoires). Ainsi, au total 144 producteurs ont été enquêtés (Tableau 1). Un critère d'âge de sélection des producteurs fixé à 40 ans a permis de s'assurer que les stratégies développées font suite à une perception effective des changements.

Les données ont été collectées par des enquêtes à travers des entretiens structurés, semi structurés et non structurés. Elles sont qualitatives et quantitatives. Elles ont été collectées en Avril 2018, à l'aide des questionnaires dans la zone d'étude. En effet, ces données sont relatives aux caractéristiques socioéconomiques (sexe, âge, niveau d'instruction, situation matrimoniale, accès aux crédits, etc.), les perceptions des riziculteurs du changement climatique, les stratégies d'adaptation aux changements climatiques, etc. 
Tableau 3. Structure De L'échantillonnage

\begin{tabular}{lllll}
\hline Villages & Malanville & Glazoué & Matéri & Total \\
\hline Agouagon & 0 & 16 & 0 & 16 \\
Magoumi & 0 & 16 & 0 & 16 \\
Zaffé & 0 & 16 & 0 & 16 \\
Madécali & 16 & 0 & 0 & 16 \\
Kotchi & 16 & 0 & 0 & 16 \\
Toumboutou & 16 & 0 & 0 & 16 \\
Kakini-Séri & 0 & 0 & 16 & 16 \\
Holli & 0 & 0 & 16 & 16 \\
Dassari & 0 & 0 & 16 & 16 \\
Total & 48 & 48 & 48 & 144 \\
\hline
\end{tabular}

Source : enquête de terrain, Avril 2018

\section{Analyse des données}

Les stratégies d'adaptation aux changements climatiques en riziculture ont été caractérisées en utilisant l'Analyse Factorielle des Correspondances Multiple (AFCM) et leurs déterminants ont été identifiés par le modèle de régression multinomial logistique.

L'AFCM inter-classes est définie par l'AFC de $X_{(k . j)}^{+}$, accompagnée de la projection en éléments supplémentaires des ixk lignes de $X_{(i k j)}$, pour représenter l'écart des profils des ixk lignes à leur moyenne. Elle maximise la variance des centres de gravité des blocs. L'AFCM intra-classes est l'AFCM de $X_{(i k j)}^{-}$qui maximise la moyenne de la variance interne aux blocs. Dans l'exemple de Drouet, les profils de mortalité $j$ des différentes classes d'âge $i$ d'une même année $k$ diffèrent beaucoup plus entre eux que ne diffère le profil d'une même classe d'âge au cours du temps. Dans ce cas précis, seule l'analyse intra-classes traduira les différences entre lignes homologues (Escofier et al, 1983 Escofier et al, 1988b). Une Classification Ascendante Hiérarchique (CAH) nous a permis ensuite de regrouper par un dendrogramme (en classes) les stratégies d'adaptation suivant leur proximité. Le choix des critères de classification a porté sur une gamme de variables de structure et de fonctionnement tirées des données collectées (stratégies d'adaptation, etc.). Ce modèle a fait preuve dans plusieurs études (Katé et al., 2014 ; Labiyi, 2017). Une fois la typologie des stratégies d'adaptation effectuée, il est question d'identifier les facteurs déterminants de ces catégories de stratégies. Ainsi, trois modèles sont utilisés dans les études des déterminants d'adoption en milieu paysan. Ils sont entre autres probit, logit et tobit. Les modèles Probit et Logit sont utilisés lorsque la variable dépendante est dichotomique et Tobit est employé à la fois pour la modélisation de la décision d'adoption et à la détermination de l'intensité d'utilisation de la technologie (Assouma et al., 2019b). Pour des raisons de commodité, le modèle Logit est le plus souvent utilisé dans la modélisation des décisions d'adoption de nouvelle technologie. En effet, les approches analytiques couramment utilisées dans une étude de 
décision d'adoption impliquant les choix multiples sont les modèles multitomial logit (MNL) et multinomial probit (MNP). Les deux modèles sont importants pour l'analyse des décisions d'adaptation des agriculteurs. Ces approches sont également appropriées pour évaluer des combinaisons alternatives de stratégies d'adaptation, y compris des stratégies individuelles (Hausman \& Wise, 1978). L'utilisation d'un modèle logit MNL pour analyser les déterminants des décisions des agriculteurs dans des études de décision d'adoption impliquant des choix multiples et plus facile à calculer que son alternative, le MNP. L'avantage d'utiliser un modèle MNL est sa simplicité dans le calcul du choix probabilités exprimables sous forme analytique (Tse, 1987). Ce modèle fournit une forme fermée pratique pour les probabilités de choix sous-jacentes, sans avoir besoin de l'intégration la méthode multivariée, ce qui facilite le calcul des situations de choix caractérisées par de nombreuses alternatives. C'est ainsi que dans le cadre de cette étude un modèle de régression multinomial logistique est utilisé.

L'équation de régression est linéaire simple et s'écrit avec la présentation des différentes modalités des variables.

$y=b_{0}+b_{1} x_{1}+b_{2} x_{2}+\ldots .+b_{6} x_{6}+b_{u}$

$b_{u}:$ constante

$x_{1}$ : sexe : $0=$ femme, $1=$ hommes

$x_{2}$ : accès au crédit : $0=$ non, $1=$ oui

$x_{3}$ : contact avec projet sur changement climatique : $0=$ non, $1=$ oui

$x_{4}$ : utilisation de l'information de prévision climatique : $0=$ non, $1=$ oui

$x_{5}$ : utilisation de la fumure organique : $0=$ non, $1=$ oui

$x_{6}$ : information sur les prévisions climatiques : $0=$ non, $1=$ oui

$x_{7}$ : alphabétisation : $0=$ non, $1=$ oui

$b_{u}:$ erreur

\section{Resultats}

L'analyse des phénomènes de variabilités climatiques ne peut se faire sans chercher à savoir si les populations locales appréhendent à leur niveau l'état du climat. Cette perception de l'évolution ou non, ou des modifications au niveau du climat, est étudiée à travers les discours des producteurs relatifs aux risques climatiques et à leurs différentes manifestations.

\section{Perception des changements climatiques dans l'agriculture et dans la riziculture}

$\mathrm{Au}$ centre et au nord du Bénin, tous les riziculteurs perçoivent clairement les effets du changement climatique sur les précipitations des pluies et sur la fertilité des sols. Ces producteurs avouent avoir adopté au moins une des stratégies nécessaires pour s'adapter. Environ 44,5\% des riziculteurs enquêtés ont remarqué un retard dans le démarrage des pluies, suivi de 
l'inondation constatée par $16,7 \%$ des producteurs. Ensuite $12 \%$ des riziculteurs ont constaté une mauvaise répartition des pluies $(\mathrm{P}<0,001)$. Près de $80 \%$ des riziculteurs ont constaté une poche de sècheresse et précocité dans le démarrage des pluies au cours de ces dix dernières années. L'arrêt précoce des pluies est moins perçu par les riziculteurs $(6 \%)$.

L'analyse par zone agro-écologique révèle que dans la commune Malanville (zone1) les riziculteurs perçoivent plus, le retard des pluies $(54,2 \%)$, alors qu'à Matéri (zone 4) et Glazoué (zone 5) peu des riziculteurs l'ont perçu. L'excès des pluies/inondation est perçue par la majorité des riziculteurs de Malanville $(31,3 \%)$ car cette commune se trouve au bord du fleuve Niger et y abrite le fleuve Sota qui déborde chaque année. Dans les deux autres communes, peu des riziculteurs ont été victime de l'inondation (tableau 2).

Le constat du changement climatique étant établi avec les riziculteurs, il importe d'évaluer l'impact des modifications de ces facteurs climatiques sur la culture du riz pluvial. Les résultats du tableau 2 indiquent la perception qu'ont les riziculteurs de l'effet des changements climatiques sur la production du riz. La plupart des riziculteurs estiment que le changement climatique a un effet négatif sur la production du riz pluvial. En effet, plus des trois-quarts des riziculteurs estiment que les rendements ont baissés avec une mauvaise levée $(73,5 \%)$ qui nécessite assez de repiquage et $81,8 \%$ estiment que les rendements ont baissé.

Tableau 2. Perception des riziculteurs de l'effet des changements climatiques sur l'agriculture et la production du riz

\begin{tabular}{|c|c|c|c|c|c|c|}
\hline \multirow[t]{2}{*}{ Variables } & \multirow[t]{2}{*}{ Perceptions } & \multicolumn{5}{|c|}{ Zone d'étude (\%) } \\
\hline & & Malanville & Matéri & Glazoué & Total & Test de Khi-deux \\
\hline \multirow[t]{8}{*}{ Agriculture } & $\begin{array}{l}\text { Perception des effets du } \\
\text { changement climatiques }\end{array}$ & 100 & 100 & 97,8 & 99,3 & $\begin{aligned} \chi^{2} & =2,12 ; \mathrm{ddl}= \\
2 ; \mathrm{P} & =0,34\end{aligned}$ \\
\hline & $\begin{array}{l}\text { Perception effet négatif cc sur } \\
\text { fertilité sol }\end{array}$ & 87,2 & 98 & 89,1 & 91,5 & $\begin{array}{l}\chi^{2}=4,08 ; \mathrm{ddl}=2 ; \\
\mathrm{P}=0,13\end{array}$ \\
\hline & $\begin{array}{l}\text { Excès } \\
\text { pluviométriques/inondations }\end{array}$ & 31,3 & 16,7 & 2,1 & 16,7 & $\begin{array}{l}\chi^{2}=1,64 ; \\
\mathrm{ddl}=2 ; \mathrm{P}=0,43\end{array}$ \\
\hline & $\begin{array}{l}\text { Retard dans le démarrage des } \\
\text { pluies }\end{array}$ & 54,2 & 43,8 & 35,4 & 44,5 & $\begin{array}{l}\chi^{2}=22,65 ; \quad \text { ddl }=2 ; \\
\mathrm{P}<0,001\end{array}$ \\
\hline & $\begin{array}{l}\text { Précocité de la saison des } \\
\text { pluies }\end{array}$ & 14,6 & 14,6 & 8,3 & 12,5 & $\begin{array}{l}\chi^{2}=0,50 ; \\
\text { ddl }=2 ; P=0,77\end{array}$ \\
\hline & $\begin{array}{l}\text { Rupture des pluies en pleine } \\
\text { saison pluvieuse (poche de } \\
\text { sécheresse) }\end{array}$ & 70,2 & 85,7 & 82,6 & 79,6 & $\begin{array}{l}\chi^{2}=3,93 \\
\text { ddl }=2 ; P=0,14\end{array}$ \\
\hline & $\begin{array}{l}\text { Mauvaise répartition des } \\
\text { pluies }\end{array}$ & 2,1 & 16,7 & 16,7 & 11,8 & $\begin{array}{l}\chi^{2}=11,78 ; \\
\mathrm{ddl}=2 ; \mathrm{P}=0,01\end{array}$ \\
\hline & Arrêt précoce des pluies & 8,3 & 5,6 & 4,2 & 6 & $\begin{array}{l}\chi^{2}=2,08 \\
\mathrm{ddl}=2 ; \mathrm{P}=0,11\end{array}$ \\
\hline \multirow[t]{2}{*}{ Riziculture } & $\begin{array}{l}\text { Perception effet négatif cc sur } \\
\text { production riz }\end{array}$ & 100 & 100 & 95,7 & 98,6 & $\begin{array}{l}\chi^{2}=4,27 ; \\
\text { ddl }=2 ; P=0,72\end{array}$ \\
\hline & Mauvaise levée & 79,2 & 77,6 & 63,8 & 73,5 & $\begin{array}{l}\chi^{2}=3,75 ; \\
\mathrm{ddl}=2 ; P=0,15\end{array}$ \\
\hline
\end{tabular}




\begin{tabular}{|c|c|c|c|c|c|}
\hline Nécessité de repiquage & 21,3 & 56 & 52,2 & 43,4 & $\begin{array}{l}\chi^{2}=14,04 ; \quad \text { ddl }=2 ; \\
\mathrm{P}=0,001\end{array}$ \\
\hline Pertes partielles récoltes & $72,3 \%$ & $80,0 \%$ & $54,3 \%$ & $\begin{array}{l}69,2 \\
\%\end{array}$ & $\begin{array}{l}\chi^{2}=7,71 ; \quad \mathrm{ddl}= \\
\mathrm{P}=0,02\end{array}$ \\
\hline Baisse rendement & $72,3 \%$ & $89,1 \%$ & $84,0 \%$ & $\begin{array}{l}81,8 \\
\%\end{array}$ & $\begin{array}{l}\chi^{2}=4,65 \\
P=0,09\end{array}$ \\
\hline $\begin{array}{l}\text { Riz sèche sur pied avant } \\
\text { épiaison }\end{array}$ & $36,2 \%$ & $40,8 \%$ & $39,1 \%$ & $\begin{array}{l}38,7 \\
\%\end{array}$ & $\begin{array}{l}\chi^{2}=0,22 \\
\mathrm{P}=0,89\end{array}$ \\
\hline Augmentation infestation & $21,3 \%$ & $38,0 \%$ & $39,1 \%$ & $\begin{array}{l}32,9 \\
\%\end{array}$ & $\begin{array}{l}\chi^{2}=4,27 \\
P=0,11\end{array}$ \\
\hline Baisse qualité paddy & $55,3 \%$ & $62,0 \%$ & $60,9 \%$ & $\begin{array}{l}59,4 \\
\%\end{array}$ & $\begin{array}{l}\chi^{2}=0,50 \\
P=0,77\end{array}$ \\
\hline Faible tallage & $31,9 \%$ & $40,0 \%$ & $45,7 \%$ & $\begin{array}{l}39,2 \\
\%\end{array}$ & $\begin{array}{l}\chi^{2}=1,86 ; \quad \text { ddl }=2 ; \\
P=0,39\end{array}$ \\
\hline Apparition tardive panicules & 22,9 & 34,7 & 46,8 & 34,7 & $\begin{array}{l}\chi^{2}=16,29 ; \quad \mathrm{ddl}=2 ; \\
\mathrm{P}=0,01\end{array}$ \\
\hline
\end{tabular}

Source : Résultat d'analyse d'enquête, 2018

\section{Stratégies d'adaptation aux effets du changement climatique en riziculture pluvial au Bénin}

Les stratégies d'adaptation aux changements climatiques restent la meilleure solution pour atténuer les effets des changements climatiques dans la production agricole. Ainsi, plusieurs stratégies sont alors utilisées dans la riziculture pour atténuer les effets des variabilités climatiques au cours de ces dernières années. Pour s'adapter face aux changements perçus dans le climat, les producteurs interrogés dans la zone d'étude utilisent plusieurs stratégies (Tableau 3). La majorité des producteurs interrogés $(98,6 \%)$ ont changé le calendrier agricole afin de s'adapter aux changements climatiques. De même, cette stratégie semble être utilisée par tous producteurs quel que soit leur localité ou zone climatique. L'irrigation de complément est une stratégie d'adaptation utilisée par $27,3 \%$ des riziculteurs dans la zone d'étude. Cependant, seuls les riziculteurs de la commune de Malanville (environ 83\%) l'utilisent comme stratégie d'adaptation.

Quant à la diversification des cultures, elle est pratiquée par $61,5 \%$ des riziculteurs comme une stratégie d'adaptation aux changements climatiques. Cette option est utilisée dans toute la région d'étude. Près de $64 \%$ des enquêtés utilisent l'introduction de nouvelles spéculations comme option d'adaptation aux changements climatiques (tableau 3). L'accroissement des superficies allouées aux cultures est une option envisagée par plus d'un quart des riziculteurs. Le repiquage de jeunes plants est une option d'adaptation envisagée par 23,1\% des riziculteurs. Cette option est plus utilisée chez les riziculteurs de la commune de Malanville (environ 53,2\%) que dans les autres zones de l'étude. La rotation de culture est une stratégie d'adaptation utilisée par environ un tiers des riziculteurs. De même, cette stratégie est utilisée par tous les riziculteurs quel que soit leur localité ou zone climatique. 
Tableau 4. Stratégies d'adaptation aux changements climatiques pratiquées par les riziculteurs au Nord Bénin

\begin{tabular}{|c|c|c|c|c|c|}
\hline \multirow[t]{2}{*}{ Stratégies d'adaptation } & \multicolumn{4}{|c|}{ Zones d'étude } & \multirow[t]{2}{*}{ Test de Khi-deux } \\
\hline & Malanville & Glazoué & Matéri & Total & \\
\hline Diversification des cultures & $55,3 \%$ & $67,4 \%$ & $62 \%$ & $61,5 \%$ & $\begin{array}{l}\chi^{2}=1,43 ; \mathrm{ddl}=2 ; \mathrm{P}- \\
\text { valeur }=0,48\end{array}$ \\
\hline $\begin{array}{l}\text { Introduction de nouvelles } \\
\text { spéculations }\end{array}$ & $60,9 \%$ & $67,4 \%$ & $64 \%$ & $64,1 \%$ & $\begin{array}{l}\chi^{2}=0,42 ; \mathrm{ddl}=2 ; \\
\text { P-valeur }=0,80\end{array}$ \\
\hline $\begin{array}{l}\text { Utilisation des informations } \\
\text { de prévision cc }\end{array}$ & $23,4 \%$ & $30,4 \%$ & $20 \%$ & $24,5 \%$ & $\begin{array}{l}\chi^{2}=1,45 ; \mathrm{ddl}=2 ; \\
\mathrm{P} \text {-valeur }=0,48\end{array}$ \\
\hline $\begin{array}{lll}\text { Utilisation } & \text { des } & \text { variétés } \\
\text { résistantes } & & \\
\end{array}$ & $17,4 \%$ & $26,1 \%$ & $28 \%$ & $23,9 \%$ & $\begin{array}{l}\chi^{2}=1,65 ; \mathrm{ddl}=2 ; \\
\mathrm{P} \text {-valeur }=0,43\end{array}$ \\
\hline $\begin{array}{l}\text { Utilisation semences } \\
\text { améliorées IR841 }\end{array}$ & $34,0 \%$ & $35,6 \%$ & $28 \%$ & $32,4 \%$ & $\begin{array}{l}\chi^{2}=0,70 ; \mathrm{ddl}=2 ; \\
\mathrm{P} \text {-valeur }=0,70\end{array}$ \\
\hline $\begin{array}{l}\text { Utilisation des variétés à } \\
\text { cycle court }\end{array}$ & $34,0 \%$ & $23,9 \%$ & $38 \%$ & $32,2 \%$ & $\begin{array}{l}\chi^{2}=2,29 ; \mathrm{ddl}=2 ; \\
\mathrm{P} \text {-valeur }=0,31\end{array}$ \\
\hline $\begin{array}{l}\text { Abandon des variétés à } \\
\text { cycle court }\end{array}$ & $2,8 \%$ & $2,8 \%$ & $1,4 \%$ & $6,9 \%$ & $\begin{array}{l}\chi^{2}=1,142 ; \mathrm{ddl}=2 ; \\
\mathrm{P} \text {-valeur }=0,76\end{array}$ \\
\hline $\begin{array}{l}\text { Augmentation du nombre de } \\
\text { graines par poquet }\end{array}$ & $12,8 \%$ & $35,6 \%$ & $26 \%$ & $24,6 \%$ & $\begin{array}{l}\chi^{2}=6,50 ; \text { ddl }=2 ; \\
\text { P-valeur }=0,03\end{array}$ \\
\hline Semis direct & $8,5 \%$ & $69,6 \%$ & $55,1 \%$ & $44,4 \%$ & $\begin{array}{l}\chi^{2}=38,60 ; \mathrm{ddl}=2 \\
; \mathrm{P} \text {-valeur } 0,001\end{array}$ \\
\hline Rotation culturale & $40,4 \%$ & $42,2 \%$ & $36 \%$ & $39,4 \%$ & $\begin{array}{l}\chi^{2}=0,41 ; \mathrm{ddl}=2 ; \\
\mathrm{P} \text {-valeur }=0,81\end{array}$ \\
\hline Réduction des superficies & $19,1 \%$ & $32,6 \%$ & $18,0 \%$ & $23,1 \%$ & $\begin{array}{l}\chi^{2}=3,48 ; \mathrm{ddl}=2 ; \\
\mathrm{P} \text {-valeur }=0,175\end{array}$ \\
\hline $\begin{array}{ll}\text { Accroissement } & \text { des } \\
\text { superficies cultivées } & \end{array}$ & $34 \%$ & $15,6 \%$ & $36 \%$ & $28,9 \%$ & $\begin{array}{l}\chi^{2}=5,73 ; \mathrm{ddl}=2 ; \\
\text { P-valeur }=0,05\end{array}$ \\
\hline $\begin{array}{l}\text { Migration agriculture vers } \\
\text { activités non agricole }\end{array}$ & $4,3 \%$ & $20 \%$ & $2,1 \%$ & $8,6 \%$ & $\begin{array}{l}\chi^{2}=11,19 ; \mathrm{ddl}=2 \\
; \mathrm{P} \text {-valeur }=0,004\end{array}$ \\
\hline $\begin{array}{l}\text { Abandon de certaines } \\
\text { spéculations }\end{array}$ & $8,5 \%$ & $15,9 \%$ & $4 \%$ & $9,2 \%$ & $\begin{array}{l}\chi^{2}=4,00 ; \text { ddl }=2 \\
\text { P-valeur }=0,13\end{array}$ \\
\hline $\begin{array}{l}\text { Plus grande utilisation } \\
\text { d'engrais chimique }\end{array}$ & $34,0 \%$ & $21,7 \%$ & $30 \%$ & $28,7 \%$ & $\begin{array}{l}\chi^{2}=1,78 ; \mathrm{ddl}=2 ; \\
\text { P-valeur }=0,40\end{array}$ \\
\hline $\begin{array}{l}\text { Intensification contrôle des } \\
\text { ravageurs }\end{array}$ & $31,9 \%$ & $19,6 \%$ & $6 \%$ & $18,9 \%$ & $\begin{array}{l}\chi^{2}=10,64 ; \mathrm{ddl}=2 \\
; \mathrm{P} \text {-valeur }=0,005\end{array}$ \\
\hline $\begin{array}{l}\text { Mise en œuvre du Système } \\
\text { Riziculture Intensive }\end{array}$ & $23,4 \%$ & $8,7 \%$ & $0 \%$ & $10,5 \%$ & $\begin{array}{l}\chi^{2}=14,36 ; \mathrm{ddl}=2 \\
; \mathrm{P} \text {-valeur }=0,001\end{array}$ \\
\hline 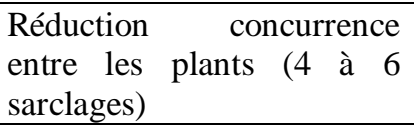 & $2,2 \%$ & $13,0 \%$ & $2 \%$ & $5,6 \%$ & $\begin{array}{l}\chi^{2}=7,02 ; \mathrm{ddl}=2 ; \\
\mathrm{P} \text {-valeur }=0,03\end{array}$ \\
\hline $\begin{array}{l}\text { Utilisation de la matière } \\
\text { organique }\end{array}$ & $34,0 \%$ & $6,5 \%$ & $14 \%$ & $18,2 \%$ & $\begin{array}{l}\chi^{2}=12,74 ; \mathrm{ddl}=2 \\
; \mathrm{P}-\text { valeur }=0,002\end{array}$ \\
\hline $\begin{array}{l}\text { Repiquage de jeunes plants } \\
\text { (1 à } 2 \text { feuilles) }\end{array}$ & $53,2 \%$ & $15,2 \%$ & $2 \%$ & $23,1 \%$ & $\begin{array}{l}\chi^{2}=38,12 ; \mathrm{ddl}=2 \\
; \mathrm{P} \text {-valeur }=0,000\end{array}$ \\
\hline $\begin{array}{l}\text { Canal drainage eau évitant } \\
\text { inondation }\end{array}$ & $38,3 \%$ & $19,6 \%$ & $4 \%$ & $20,3 \%$ & $\begin{array}{l}\chi^{2}=17,64 ; \mathrm{ddl}=2 \\
; \mathrm{P} \text {-valeur }=0,000\end{array}$ \\
\hline
\end{tabular}




\begin{tabular}{|c|c|c|c|c|c|}
\hline $\begin{array}{l}\text { Irrigation de complément } \\
\text { (motopompe) }\end{array}$ & $83 \%$ & $0 \%$ & $0 \%$ & $27,3 \%$ & $\begin{array}{l}\chi^{2}=109,53 ; \mathrm{ddl}=2 \\
; \mathrm{P} \text {-valeur }=0,000\end{array}$ \\
\hline Semis sur un sol sec & $2,1 \%$ & $0 \%$ & $4 \%$ & $2,1 \%$ & $\begin{array}{l}\chi^{2}=1,86 ; \mathrm{ddl}=2 ; \\
\text { P-valeur }=0,39\end{array}$ \\
\hline $\begin{array}{l}\text { Prière ou rituels } \\
\text { traditionnels }\end{array}$ & $19,1 \%$ & $15,2 \%$ & $28 \%$ & $21 \%$ & $\begin{array}{l}\chi^{2}=2,50 ; \mathrm{ddl}=2 ; \\
\text { P-valeur }=0,28\end{array}$ \\
\hline $\begin{array}{l}\text { Actions de lutte antiérosive } \\
\text { (cordons pierreux, bande } \\
\text { enherbée, etc.) }\end{array}$ & $38,3 \%$ & $15,2 \%$ & $10 \%$ & $21 \%$ & $\begin{array}{l}\chi^{2}=13,06 ; \mathrm{ddl}=2 \\
; \mathrm{P} \text {-valeur }=0,001\end{array}$ \\
\hline Utilisation des épouvantails & $66 \%$ & $63 \%$ & $48 \%$ & $58,7 \%$ & $\begin{array}{l}\chi^{2}=3,74 ; \mathrm{ddl}=2 ; \\
\text { P-valeur }=0,15\end{array}$ \\
\hline $\begin{array}{l}\text { Paillage en utilisant des } \\
\text { résidus }\end{array}$ & $8,7 \%$ & $10,9 \%$ & $4,3 \%$ & $7,9 \%$ & $\begin{array}{l}\chi^{2}=1,45 ; \mathrm{ddl}=2 ; \\
\text { P-valeur }=0,48\end{array}$ \\
\hline $\begin{array}{l}\text { Assurance contre les dégâts } \\
\text { des ravageurs et cc }\end{array}$ & $0 \%$ & $2,2 \%$ & $0 \%$ & $0,7 \%$ & $\begin{array}{l}\chi^{2}=2,12 ; \mathrm{ddl}=2 ; \\
\text { P-valeur }=0,34\end{array}$ \\
\hline Réalisation des diguettes & $0 \%$ & $5,9 \%$ & $5,6 \%$ & $3,8 \%$ & $\begin{array}{l}\chi^{2}=1,01 ; \mathrm{ddl}=2 ; \\
\text { P-valeur }=0,60\end{array}$ \\
\hline
\end{tabular}

Source : Résultat d'analyse d'enquête, 2018

\section{Typologie des stratégies d'adaptation aux changements climatiques en riziculture au Nord Bénin}

Différentes typologies de stratégie d'adaptation ont été réalisées au cours des deux dernières décennies, avec récemment des détails plus précis émergeant. Smit et al. (2000) avaient montré que les typologies d'adaptation existantes se focalisent sur cinq domaines principaux par rapport : (i) au temps (anticipative, réactive), (ii) à l'intention (autonome, planifiée), (iii) à la répartition spatiale (locale, régionale, nationale), (iv) à la forme (technique, institutionnelle, légale, comportementale ou éducationnelle), et (v) au degré de changement nécessaire (incrémental, transformationnel) (Carter et al., 1994, Fidelman et al., 2013, Huq et al., 2003; Smit et Skinner, 2002; Smit et Wandel, 2006; Wilbanks et Kates, 1999).

\section{Caractérisation des stratégies}

\section{Le choix du nombre d'axes factoriels}

Le choix du nombre se fait à l'aide du test de Coud ou Scree Plot. En effet, à travers l'observation de cette courbe, on détecte les composantes qui ont un indice de valeur propre supérieure ou égale à 1 . On décèle alors trois axes factoriels pour l'AFCM. 


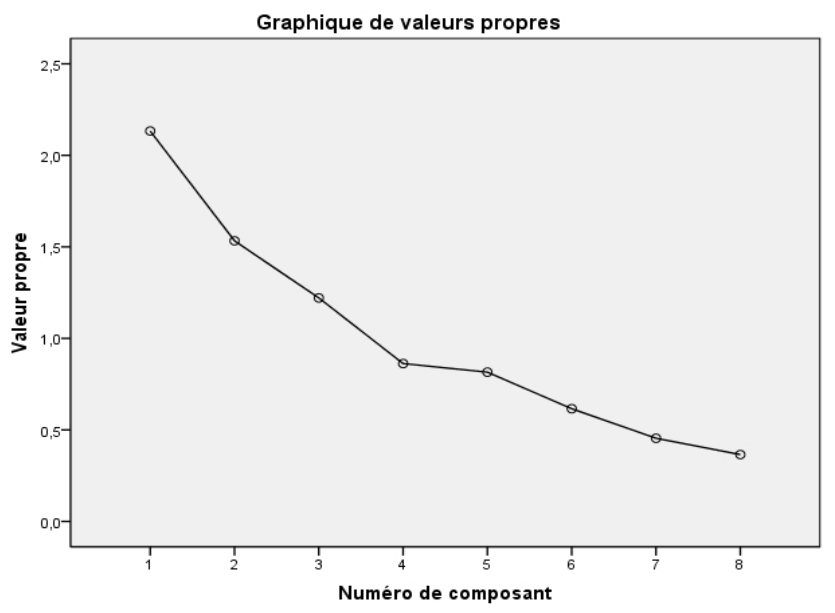

Figure 2. Le test de Coud

Source : Résultat d'analyse d'enquête, 2018

Le traitement des variables introduites dans la classification a été rendu possible grâce à une Analyse Factorielle de Correspondances Multiples (AFCM). Le choix de ce traitement a été fait compte tenu de la nature (hétérogène) de certaines variables à apporter les mêmes informations et de leur taille dans l'analyse faite. Ainsi, il ressort des données du tableau 4 et de l'analyse effectuée sur les 144 individus, que les deux (02) premiers axes factoriels permettent d'expliquer à 50,78\% les informations obtenues. Donc, les $49,22 \%$ des informations restantes sont dues aux variables qui n'ont pas été prises en compte dans l'analyse.

Tableau 4. Contribution cumulée à l'inertie totale des deux premiers axes factoriels de

\begin{tabular}{lll}
\multicolumn{3}{c}{ l'AFCM } \\
\hline Axe factoriels & \% d'inertie & \% cumulé \\
\hline 1 & 29,35 & 29,35 \\
2 & 21,43 & 50,78 \\
\hline
\end{tabular}

Source : Résultat d'analyse d'enquête, 2018

En effet, la définition de chaque axe factoriel a été faite sur la base de l'étude de la contribution relative des modalités des variables à l'inertie expliquée par les axes et se présente comme suit (Tableau 5). L'analyse compte après apurement 16 modalités actives. Pour chaque axe, le pourcentage d'inertie théorique moyen expliqué par chaque modalité est de 6,25\% (100\%/16). Nous caractérisons l'axe 1 à l'aide des modalités ayant une contribution supérieure à 6,25 . Par exemple, si la modalité «Non» de la diversification des cultures se retrouvait du côté négatif de l'axe 1 (coordonnée négative), on pourrait s'attendre à ce que les individus possédant les modalités actives bien représentées du côté négatif de l'axe 1, aient plus de chance d'avoir comme modalité « Oui » pour la diversification des cultures. 
Tableau 5. Définition des axes factoriels pour les espèces pêchées

\begin{tabular}{|c|c|}
\hline $\begin{array}{l}\text { Axe } \\
\text { factoriels }\end{array}$ & Axe négatif \\
\hline \multirow{6}{*}{1} & $\begin{array}{l}\text { Introduisez-vous de nouvelles spéculations dans les systèmes de } \\
\text { production (NVCULT) ? }\end{array}$ \\
\hline & NVCULT_Non $\quad$ NVCULT_Oui \\
\hline & Diversifiez-vous vos cultures (DICUL) ? \\
\hline & DICUL_Non $\quad$ DICUL_Oui \\
\hline & Faites-vous des semis direct (SEMIDIR)? \\
\hline & $\begin{array}{ll}\text { SEMIDIR_Oui } & \text { SEMIDIR_Non } \\
\end{array}$ \\
\hline \multirow{10}{*}{2} & Pratiquez-vous la rotation culturale (ROTCULT) ? \\
\hline & $\begin{array}{ll}\text { ROTCULT_Oui } & \text { ROTCULT_Non } \\
\end{array}$ \\
\hline & Augmentez-vous (Accroissement) les superficies cultivées (ACCSUP) ? \\
\hline & $\begin{array}{ll}\text { ACCSUP_Oui } & \text { ACCSUP_Non } \\
\end{array}$ \\
\hline & $\begin{array}{l}\text { Utilisez-vous plus d'engrais chimique avec une quantité élevée } \\
\text { (PLUSENGCH)? }\end{array}$ \\
\hline & $\begin{array}{ll}\text { PLUSENGCH_Oui } & \text { PLUSENGCH_Non }\end{array}$ \\
\hline & Utilisez-vous des variétés résistantes (VARRESIT) ? \\
\hline & $\begin{array}{ll}\text { VARRESIT_Non } & \text { VARRESIT_Oui } \\
\end{array}$ \\
\hline & Utilisez-vous des variétés à cycle court (UTILVACYC) ? \\
\hline & $\begin{array}{ll}\text { UTILVACYC_Non } & \text { UTILVACYC_Oui }\end{array}$ \\
\hline
\end{tabular}

Source : Résultat d'analyse d'enquête, 2018

La figure 3 montre la représentation graphique du regroupement des riziculteurs en fonction des stratégies d'adaptation adoptées dans la riziculture et leurs projections sur les deux premières dimensions. La superposition de cette figure permet d'observer trois regroupements des riziculteurs repartis de façon homogène. Ces regroupements permettent une meilleure classification des stratégies d'adaptation dans la riziculture ressortis par l'AFCM. De cette figure, il ressort trois groupes de stratégies d'adaptation qui sont constitués. Les caractéristiques générales de chacune des classes de riziculteurs en fonction des stratégies d'adaptation aux changements climatiques se présentent comme suit :

- Classe 1 (cluster 1), tout ce qui est mieux représenté concerne le groupe formé par: Rotations culturales, Accroissement des superficies cultivées, Plus grande utilisation d'engrais chimique et Utilisation des variétés résistantes.

- Classe 2 (cluster 2), les variables les plus représentées forment un groupe dont la configuration est la suivante : semis direct et utilisation des variétés de cycle court

- Classe 3 (cluster 3) est représentée par les variables : diversification culturales et introduction de nouvelles cultures. 


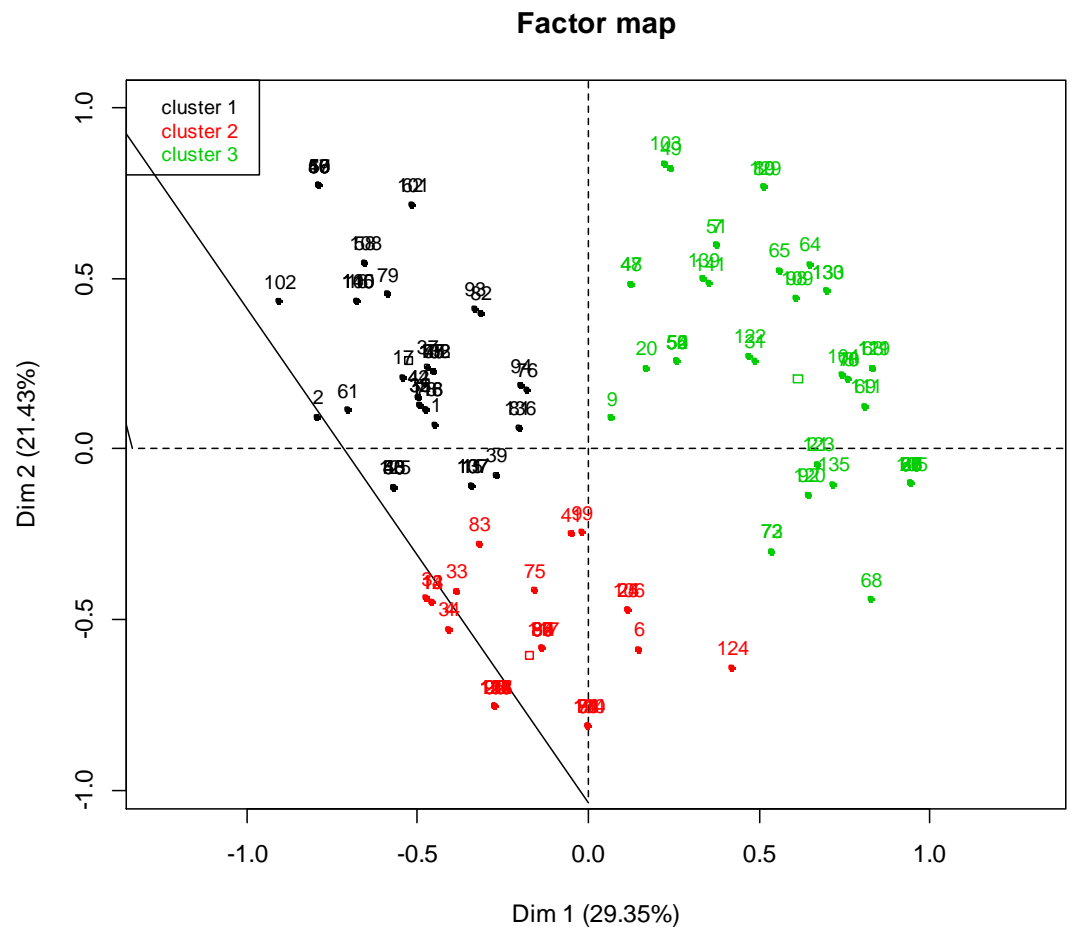

Figure 3. Représentation graphique des regroupements issus de l'AFCM Source : Résultat d'analyse d'enquête, 2018

\section{Identification et description des différentes classes Identification des classes}

Pour la classification hiérarchique des stratégies d'adaptation, nous avons choisi initialement un indice de niveau qui correspond à trois (03) classes. Avec le Logiciel R, la méthode de liaison et la mesure de distance choisies influencent considérablement les résultats du regroupement des variables. Cette Classification Ascendante Hiérarchique (CAH) a permis d'identifier trois (03) groupes de producteurs qui diffèrent selon les stratégies adoptées (Figure 4). 
Hierarchical Clustering
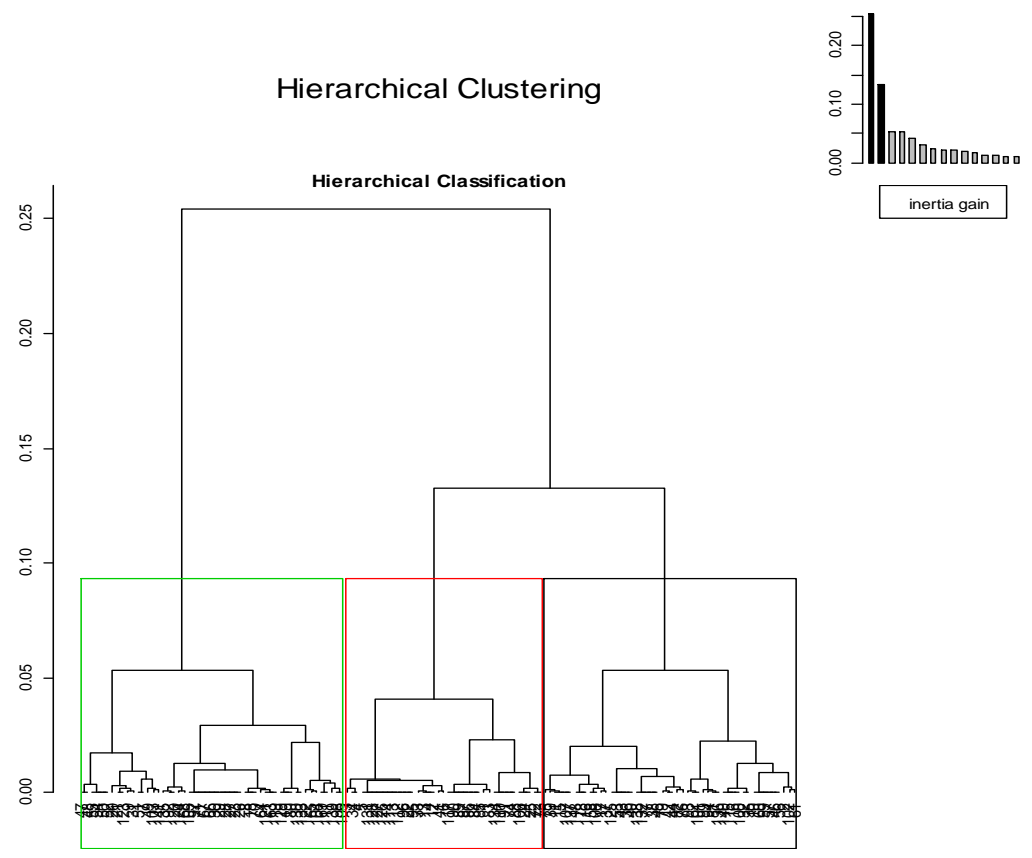

Figure 4. Dendrogramme de la classification hiérarchique Description des différents systèmes

Après avoir fait la classification en trois classes, à l'aide des résultats de la classification (Tableau 6) donnant respectivement la répartition des classes en effectif et les caractéristiques générales des producteurs en fonction des différentes variables utilisées.

\section{Description de la classe 1}

Les producteurs de la classe 1 adoptent les stratégies de rotations culturales $(28,7 \%)$, accroissement des superficies cultivées (20,6\%), plus grande utilisation d'engrais chimique $(17,9 \%)$ et Utilisation des variétés résistantes $(16,9 \%)$. Ces producteurs représentent $34 \%$ de la taille totale de l'échantillon. Ils utilisent en moyenne 160,92 $( \pm 231,84) \mathrm{kg}$ de NPK et 189,72 $( \pm 300,94) \mathrm{kg}$ d'urée sur la superficie totale du riz emblavée qui est de 1,60 $( \pm 1,33)$ ha. Ils ont obtenu un rendement moyen de $4601,63( \pm 6$ 932,22) kg avec un revenu net de $469063,46( \pm 181153,11)$ FCFA sur toute la production du riz de l'exploitation agricole.

\section{Description de la classe 2}

Les producteurs appartenant à cette classe pratique le semis direct (31\%) et utilisation des variétés de cycle court (20\%). Ils appliquent en moyenne $161,76( \pm 345,23) \mathrm{Kg}$ de NPK et $162,35( \pm 413,10) \mathrm{Kg}$ de l'urée et ont un revenu moyen net de 698 897,32 $( \pm 195667,72)$ FCFA avec un rendement 5 718,33 $( \pm 14694,37) \mathrm{Kg}$ sur une superficie rizicole moyenne de 
$1,76( \pm 1,91)$ ha. Par ailleurs, les individus de cette classe représentent $29,2 \%$ de la taille de l'échantillon totale soit un effectif de 42 riziculteurs.

\section{Description de la classe 3}

Ici, les producteurs pratiquent la diversification culturales $(31,2 \%)$ et l'introduction de nouvelles $(38,5 \%)$. Les individus de cette classe sont 53 riziculteurs soit $36,8 \%$ de la taille de l'échantillon. Ils emblavent une superficie de $1,64( \pm 1,53)$ ha sur laquelle ils appliquent une dose de 144,56 $( \pm 290,09) \mathrm{Kg}$ d'urée et $175,03( \pm 301,297) \mathrm{Kg}$ de NPK où ils obtiennent un rendement de $3410,68(4581,90) \mathrm{Kg}$ avec un revenu net $321221,93( \pm 174$ 183,05) FCFA.

Tableau 6. Répartition des classes en effectif

\begin{tabular}{|c|c|c|c|c|}
\hline \multirow[t]{2}{*}{ Variables } & \multicolumn{3}{|c|}{ Typologie des producteurs } & \multirow[t]{2}{*}{ Total } \\
\hline & Classe 1 & Classe 2 & Classe 3 & \\
\hline $\begin{array}{l}\text { Fréquence d'individus par classe } \\
(\%)\end{array}$ & $49(34 \%)$ & $42(29,2 \%)$ & $53(36,8 \%)$ & $144(100 \%)$ \\
\hline \multicolumn{5}{|c|}{ Stratégies d'adaptation aux changements climatiques } \\
\hline Diversification des cultures & $15,3 \%$ & $15,3 \%$ & $31,2 \%$ & $61,8 \%$ \\
\hline $\begin{array}{l}\text { Introduction de nouvelles } \\
\text { spéculations }\end{array}$ & $11,1 \%$ & $15,3 \%$ & $38,5 \%$ & $64,3 \%$ \\
\hline $\begin{array}{l}\text { Utilisation des variétés à cycle } \\
\text { court }\end{array}$ & $7,6 \%$ & $20 \%$ & $4,3 \%$ & $31,9 \%$ \\
\hline Rotation culturale & $28,7 \%$ & $5,6 \%$ & $5,6 \%$ & $39,9 \%$ \\
\hline Semis direct & $7,5 \%$ & $31,0 \%$ & $5,6 \%$ & $44,1 \%$ \\
\hline $\begin{array}{l}\text { Accroissement des superficies } \\
\text { cultivées }\end{array}$ & $20,6 \%$ & $3,5 \%$ & $4,6 \%$ & $28,7 \%$ \\
\hline $\begin{array}{l}\text { Plus grande utilisation d'engrais } \\
\text { chimique }\end{array}$ & $17,9 \%$ & $6,3 \%$ & $4,3 \%$ & $28,5 \%$ \\
\hline Utilisation des variétés résistantes & $16,9 \%$ & $2,3 \%$ & $4,6 \%$ & $23,8 \%$ \\
\hline \multicolumn{5}{|c|}{ Quantités des engrais chimiques en fonction de la typologie des riziculteurs } \\
\hline NPK en $\mathrm{Kg}$ & $\begin{array}{c}160,92 \\
( \pm 231,84) \\
\end{array}$ & $\begin{array}{c}161,76 \\
( \pm 345,23) \\
\end{array}$ & $\begin{array}{c}175,03 \\
( \pm 301,297) \\
\end{array}$ & $\begin{array}{c}166,36 \\
( \pm 292,013) \\
\end{array}$ \\
\hline Urée en $\mathrm{Kg}$ & $\begin{array}{c}189,72 \\
( \pm 300,94) \\
\end{array}$ & $\begin{array}{c}162,35 \\
( \pm 413,10) \\
\end{array}$ & $144,56( \pm 290,09)$ & $165,12( \pm 332,11)$ \\
\hline \multicolumn{5}{|c|}{ Rendements obtenus de la riziculture en fonction de la typologie des riziculteurs } \\
\hline Revenu Net en FCFA & $\begin{array}{c}469063,46 \\
( \pm 181153,11) \\
\end{array}$ & $\begin{array}{c}698897,32 \\
( \pm 195667,72) \\
\end{array}$ & $\begin{array}{c}321221,93 \\
( \pm 174183,05) \\
\end{array}$ & $\begin{array}{c}481684,44( \pm 1 \\
268452,44) \\
\end{array}$ \\
\hline Superficie du riz emblavée en ha & $1,60( \pm 1,33)$ & $1,76( \pm 1,91)$ & $1,64( \pm 1,53)$ & $1,66( \pm 1,58)$ \\
\hline Quantité du riz récoltée en $\mathrm{Kg}$ & $\begin{array}{c}4601,63( \pm 6 \\
932,22)\end{array}$ & $\begin{array}{c}5718,33( \pm 14 \\
694,37)\end{array}$ & $\begin{array}{c}3410,68( \pm 4 \\
581,90)\end{array}$ & $4489( \pm 9303,35)$ \\
\hline
\end{tabular}

Source : Résultat d'analyse d'enquête, 2018

\section{Déterminants des stratégies d'adaptation}

Nous rappelons que les variables expliquées sont représentées par les classes des stratégies d'adaptation adoptées par les riziculteurs pour atténuer les risques liés aux variabilités climatiques observées dans les systèmes de culture dans la zone d'étude. Les résultats montrent que le modèle estimé est 
globalement statistiquement significatif. En effet, le test d'indépendance de Khi-deux du modèle $\left(\chi^{2}=79,656\right)$ est significatif à $1 \%$. Cela stimule qu'il y a une forte corrélation entre les variables (Pseudo $R^{2}=0,427$ ). De plus, $A u$ niveau des paramètres estimés, on trouve globalement quatre (4) variables ayant une influence significative sur la probabilité d'adoption des stratégies d'adaptation face aux changements climatiques.

De l'analyse de tableau 7, il ressort pour la classe 1 que les facteurs déterminants des stratégies d'adaptation sont : le sexe, le contact avec un projet sur le changement climatique, l'utilisation de fumure organique et l'accès à l'information sur les prévisions climatiques. En effet, le sexe est significatif à $1 \%$. Ce facteur influence plus sur les stratégies d'adaptation que le contact avec un projet sur le changement climatique, l'utilisation de fumure organique et l'accès à l'information sur les prévisions climatiques qui sont significatifs à $10 \%$.

Pour ce qui est de la classe 2 , le contact avec un projet sur le changement climatique et l'utilisation de fumure organique sont les facteurs déterminants des stratégies d'adaptation. L'utilisation de fumure organique est le facteur important qui affecte l'adaptation au changement climatique dans cette classe. Ce facteur est significatif à 1\%. L'autre facteur qui influence les décisions d'adaptation aux changements climatiques des producteurs est le contact avec projet cc (significatif à 10\%).

Tableau 7. Résultats de la régression

\begin{tabular}{|c|c|c|c|c|}
\hline & Stratégie cla & & Stratégie cl & sse 2 \\
\hline Variables & $\boldsymbol{\beta}$ & Signification & $\boldsymbol{\beta}$ & Signification \\
\hline Constante & $-4,225 * *$ & 0,034 & $-2,341 *$ & 0,018 \\
\hline CRED & $-1,601$ & 0,201 & 0,323 & 0,613 \\
\hline SEX & $22,157 * * *$ & 0,000 & 21,677 & \\
\hline CTPROJCC & $3,857 *$ & 0,011 & $1,503 *$ & 0,029 \\
\hline UTILINFCC & $-3,561$ & 0,15 & 0,977 & 0,209 \\
\hline AFUMORG & $2,77 *$ & 0,054 & $30,27 * * *$ & 0,000 \\
\hline INFCC & $2,462 *$ & 0,06 & 0,119 & 0,874 \\
\hline INSTR & $-1,464$ & 0,173 & $-0,462$ & 0,468 \\
\hline Nombre observation & 144 & & & \\
\hline Khi-deux & 79,656 & & & \\
\hline Prob chi deux & 0,000 & & & \\
\hline Pseudo R-deux & 0,427 & & & \\
\hline
\end{tabular}

\section{Discussion}

Le changement climatique est perçu par les populations rurales du Nord-Bénin de différentes manières. Les perceptions identifiées sont conformes à celles identifiées par Katé et al. (2014) au nord-Benin dans la 
commune de Banikoara et avaient dénombré au total 24 perceptions. Yegbemey et al. (2014) avaient trouvé qu'au Nord-Bénin les producteurs du maïs percevaient le changement climatique à travers le retard dans le démarrage des pluies, les poches de sécheresse au cours de la saison pluvieuse, la mauvaise répartition spatiale des pluies, les vents violents et la chaleur excessive. Ces perceptions corroborent avec celles identifiés par la présente étude au centre et au Nord du Bénin dans la production du riz. Ces changements de prévisions climatiques induisent la baisse des rendements, de la fertilité des sols, la fréquence de repiquage des plants et 1'ajustement du calendrier agricole par la modification des dates de semis ainsi que l'irrigation de complément. Ce résultat est confirmé par les travaux de Baudoin et al. (2014) et de Amegnaglo et al. (2018). Ils sont parvenus à la conclusion selon laquelle l'agriculture irriguée n'est pas une bonne stratégie d'adaptation aux changements climatiques pour les exploitations agricoles car cette stratégie engendre des coûts très élevés en ce qui concerne l'acquisition des équipements (motopompes) créations des barrages ou forages sur les sites de production. Cette stratégie serait plus avantageuse et bénéfique pour les riziculteurs qui sont proches des fleuves ou des barrages. Cependant, seuls les riziculteurs de la commune de Malanville utilisent cette stratégie d'adaptation et cela s'explique par le fait que le fleuve Sota s'y trouve et on retrouve au niveau des parcelles de cette zone, des puits tubés d'à peine $5 \mathrm{~m}$ de profondeur qui regorgent d'eau. L'introduction de nouvelles spéculations et l'accroissement des superficies allouées aux cultures sont aussi des options d'adaptation aux changements climatiques envisagées par grand nombre des riziculteurs qui permettraient aux riziculteurs de tirer le meilleur rendement des conditions climatiques (Amegnaglo et al., 2018). Le repiquage de jeunes plants (stade 1 à 2 feuilles) est une alternative d'adaptation que certains riziculteurs $(23,1 \%)$ jugent comme solutions d'adaptation. Atidegla, et al. (2017) ont trouvé qu'au Sud-Bénin, cette stratégie n'est favorable que pour les riziculteurs qui produisent dans les bas-fonds ou dans les zones marécageuses. C'est en effet, un des principes du Système de Riziculture Intensive (SRI) qui est aussi une stratégie d'adaptation face au climat qui est adoptée par quelques producteurs $(10,5 \%)$ de Malanville et de Glazoué. La rotation de culture est également une stratégie d'adaptation utilisée par tous les riziculteurs quel que soit leur localité ou zone climatique. Par contre, Kahsay et Hansen, (2016) dans leur étude sur l'impact du changement climatique et de la politique d'adaptation sur la production agricole en Afrique de l'Est avaient trouvé que la rotation culturale avec l'utilisation continue de la même variété amplifie et favorise la résistance des parasites et des ravageurs qui déstabilisent les revenus agricoles des petits producteurs. Cette option a été identifié par d'autres études notamment celles de Baudoin et al., (2014) et Fadina et Barjolle, (2018). 
Plusieurs facteurs influencent le choix des stratégies d'adaptation au changement climatique selon la classe où elles sont classées. En effet, les stratégies de la classe 1 ont pour facteurs déterminants le sexe, le contact avec un projet sur le changement climatique, l'utilisation de fumure organique et l'accès à l'information sur les prévisions climatiques. Le sexe est plus déterminant aux stratégies car il est significatif à $1 \%$ alors que les autres sont significatifs à $10 \%$. Le contact avec projet sur le changement climatique et l'utilisation de fumure organique sont ceux qui influencent le choix des stratégies de la classe 2 . Le premier est significatif à $10 \%$ tandis que le second est significatif à $1 \%$. On conclut ainsi que l'utilisation de fumure organique est le facteur important qui affecte l'adaptation au changement climatique des stratégies de la classe 2. Ce résultat est conforme à ceux de Eisenack \& Stecker (2010) qui trouve que l'accès du public aux informations d'alerte précoce est un facteur important affectant l'adaptation au changement climatique. De même Yegbemey et al. (2019) ont trouvé la fourniture des informations météorologiques aux producteurs du Nord-Bénin par SMS leur permettent de prendre des décisions concernant l'allocation des ressources de production et aussi de connaître réellement les jours pluvieux afin de programmer les activités champêtres. Pour De Munck (2013), les hommes sont plus aptes à prendre des décisions favorisant la rentabilité de la production agricole contrairement aux femmes agricultrices en Afrique. De plus, les hommes sont les chefs d'exploitation donc toutes décisions proviennent du chef. Les producteurs ayant eu connaissance des effets positifs des engrais organiques contrairement aux engrais minéraux combinent cette stratégie aux autres. On pourrait l'expliquer par le fait que les producteurs ont acquis des informations et des résultats positifs issus des essais ce qui les a motivés à procéder ainsi. Il est à noter que des services de vulgarisations prennent en compte l'utilisation de fumures organiques et des plantes améliorantes dans la diffusion des innovations. Du fait que cette pratique revêt d'importants éléments minéraux dont leurs utilisations dans les systèmes de production n'ont aucun impact néfaste sur les aliments et l'environnement et aussi les stratégies d'adaptation approuvées par la recherche scientifique et les comités en charge d'atténuations des effets du changement climatique pour un monde sans faim.

\section{Conclusion}

Il est reconnu par la communauté scientifique que les changements climatiques existent et ont des effets notamment sur l'agriculture dans les pays en développement. Ils affectent non seulement les moyens de subsistance des populations rurales, mais la sécurité alimentaire des populations. La combinaison des stratégies d'adaptation constitue une option dans la littérature qui permet d'atténuer les effets des changements climatiques. C'est l'objectif qu'a poursuivi le présent travail, pour identifier les catégories de stratégies et 
les facteurs déterminants. L'étude à fait ressortir trois (03) catégories de stratégies qui se diffèrent par le contact des producteurs avec un projet sur le changement climatique, le niveau d'instruction, la zone agro-écologique, le sexe et les informations sur les prévisions climatiques. Le $1^{\text {er }}$ groupe de producteur pratique l'irrigation de complément et l'utilisation de la matière organique; le $2^{\text {ème }}$ groupe pratique la diversification des cultures, le semis direct et l'utilisation de semences améliorées (IR841) et le $3^{\text {ème }}$ groupe : différentes dates de semis et réduction de concurrence entre les plantes (3 à 6 sarclages). Les principaux déterminants des stratégies d'adaptation sont : le sexe, le contact avec un projet sur le changement climatique, l'utilisation de la fumure organique et l'accès à l'information sur les prévisions climatiques. Ces résultats peuvent servir de boussole pour les politiques de promotion des stratégies d'adaptation dans le contexte des changements climatiques.

\section{References:}

1. Abdou, R., Malice M., Bakasso, Y., Saadou, M. et Baudoin, J.P. 2014. Taxonomie locale et analyse des critères des paysans pour caractériser les différents écotypes d'oignons (Allium cepa L.) du Niger. Cah Agric 23 : 166-76. doi : 10.1684/agr.2014.0700

2. Alauddin et Sarker, 2014. Décisions et stratégies d'adaptation au niveau des exploitations et du changement climatique dans les régions du Bangladesh sujettes à la sécheresse et aux eaux souterraines : une étude empirique. Economie Ecologique, Volume 106, pages 204-213

3. Amegnaglo, K., Béssan, Dourma, M., Akpavi, S., Akodewou, Amah., Wala, K., Diwediga, B., Atakpama, W, A., Gbodan, K.M., Léonard, Batawila, K., et Akpagana, K. 2018. Caractérisation des formations végétales pâturées de la zone guinéenne du Togo : typologie, évaluation de la biomasse, diversité, valeur fourragère et régénération. International Journal Biological and chemical sciences. Int. J. Biol. Chem. Sci. 12(5): 2065-2084, October 2018 ISSN 1997-342X (Online), ISSN 1991-8631 (Print).

4. Arimi K. 2014. Determinants of climate change adaptation strategies used by rice farmers in Southwestern, Nigeria. Journal of Agriculture and Rural Development in the Tropics and Subtropics. Vol. 115 No. 2 (2014) 91-99

5. Arodokoun, U., Dedehouanou, H., Adeoti, R., Adegbola, P., Adekambi, S., et Katary, A. 2012. Rôle des NTIC dans l'adaptation aux changements climatiques par les producteurs de coton du CentreBénin. African Crop Science Journal, 20(2), 409-423.

6. Assouma, D. S., Ollabode, N., et Yabi, J.A. 2019b. Les Déterminants des Changements Sociaux Lies a la Gouvernance de l'eau Autour du 
Périmètre Rizicole de Malanville au Nord-est du Benin. European Scientific Journal 15 (21), 1857- 7431.

7. Assouma, D., Ollabode, N., Issaka, K., et Yabi, A.J. 2019a. Performance économique des producteurs sous divers systèmes de gestion et de distribution de l'eau dans le périmètre rizicole de Malanville. In Communication à la 1ère conférence internationale sur enjeux et perspectives économiques en Afrique Francophone à l'université Cheikh Anta Diop de Dakar, (4 au 6 février 2019).

8. Atidegla, C. S., Koumassi, H. D., Mouzou, E. T., et Houssou, E. 2017. Variabilité climatique et production du riz dans le bas-fond de Dokomey au Bénin. Journal de la Recherche Scientifique de l'Université de Lomé, 19(2), 259-276.

9. Ayedegue, L.U., Adegbola, P.Y., Yabi, A.J. 2018. Efficacité technique d'utilisation des ressources en eau et les déterminants : cas des périmètres rizicoles irrigués du Bénin. Spécial hors-série $\mathrm{n}^{\circ} 4$ Janvier 2018, et Science et technique, Sciences naturelles appliquées (319-322).

10. Below, T.B., Mutabazi, K.D., Kirschke, D., Franke, C., Sieber, S., Siebert, R., et Tscherning, K. 2012. Can Farmers' Adaptation to Climate Change Be Explained by Socio-economic Household-level Variables? Global Environmental Change22(1): 223-235

11. Boudon, R. 2004. Théorie du choix rationnel ou individualisme méthodologique ? Revue du MAUSS, $\mathrm{n}^{\mathrm{0}} 24(2)$, 281-309. doi:10.3917/rdm.024.0281.

12. Breumier, P., Ramarosandratana, A., Ramanantsoanirina, A., Vom Brocke, K., Marquié, C., Dabat, M. H., \& Raboin, L. M. 2018. Evaluation participative des impacts de la recherche sur le riz pluvial d'altitude à Madagascar de 1980 à 2015. Cahiers agricultures, 27(1).

13. Challinor, A., Watson, J., Lobell, D. Howden S.M., Smith D. R., Chhetri N. 2014. A meta-analysis of crop yield under climate change and adaptation. Nature Clim Change 4, 287-291 (2014) doi:10.1038/nclimate2153

14. Chia, E., Petit, M., et Brossier, J. 2014. Théorie du comportement adaptatif et agriculture familiale. L'agriculture en famille : travailler, réinventer, transmettre, 81 .

15. Dakossi M., 1994. Bilan de l'eau et production agricole dans la dépression de la Lama. Mémoire de maîtrise de Géographie. DGAT/UAC. 82p.

16. De Munck, C. 2013. Modélisation de la végétation urbaine et des stratégies d'adaptation au changement climatique pour l'amélioration du confort climatique et de la demande énergétique en ville (Doctoral dissertation). 
17. Deressa, T., Hassan, R. M., Alemu, T., Yesuf, M. et Ringler, C. 2008. Analyzing the Determinants of Farmers' Choice of Adaptation Methods and Perceptions of Climate Change in the Nile Basin of Ethiopia. IFPRI Discussion Paper 00798

18. Eisenack, K. et Stecker, R. 2010. An Action Theory of Adaptation to Climate Change. Berlin Conference on the Human Dimensions on Global Environmental Change.

19. Escofier, B. et Drouet, D. 1983. Analyse des différences entre plusieurs tableaux de fréquence. Les cahiers de l'analyse des données, tome 8, no 4. p. 491-499.

20. Fadina, A. M., Roland et Barjolle D. 2018. Farmers' Adaptation Strategies to Climate Change and Their Implications in the Zou Department of South Benin, Article environments MDPI

21. FAO. 2007. Codex alimentarius Commission: procedural manual. Joint FAO/WHO Food Standards Programme... - Book

22. Fidelman, P., Leitch, A. M., et Nelson, D.R. 2013. Déballer l'adaptation multiniveau au changement climatique dans la grande barrière de corail, Australie. doi.org/10.1016/j.gloenvcha.2013.02.016

23. Fussel, Hans-Martin. 2007. Adaptation Planning for Climate Change: Concepts, Assessment Approaches and Key Lessons, Article in Sustainability Science, doi: 10.1007/s11625-007-0032-y.

24. Gemenne, F., Blocher, J. M. D., De Longueville, F., Vigil Diaz Telenti, S., Zickgraf, C., Gharbaoui, D., et Ozer, P. 2017. Changement climatique, catastrophes naturelles et déplacements de populations en Afrique de l'Ouest. Geo-Eco-Trop: Revue Internationale de Géologie, de Géographie et d'Écologie Tropicales, 41(3).

25. Gnanglè, P. C., Egah, J., Baco, M. N., Gbemavo, C. D., Kakaï, R. G., et Sokpon, N. 2012. Perceptions locales du changement climatique et mesures d'adaptation dans la gestion des parcs à karité au Nord-Bénin. International Journal of Biological and Chemical Sciences, 6(1), 136149.

26. Hausman, J. et McFadden, D. 1984. Specification tests for the multinomial logit model. Econometrica 52 (5), 1219-40.

27. Hausman, J. et Wise, D. 1978. A conditional probit model for qualitative choice: Discrete decisions recognizing interdependence and heterogeneous preferences. Econometrica 46, 403-26.

28. Houssou, I. P. A., Adjovi, I. N. R. A., Dansou, I. V., HounyevouKlotoe, I. A., Hotegni, B. A. B., et Mensah, I. G. A. 2016. Guide pratique de production du riz blanc au Bénin : Aspect postrécolte. Fiche technique, Dépôt légal, (8529). 
29. Huq, S. et Adger, W. N. et Brown, K. 2003. Adaptation au changement climatique dans les pays en développement, doi.org/10.1191/1464993403ps060oa.

30. Idoma, K., Ikpe, E., Ejeh, L., et Mamman, M., 2017. Farmers Adaptation Strategies to the Effect of Climate Variation on Rice Production: Insight from Benue State, Nigeria. Environment and Ecology Research 5(4): 289-301, 2017. DOI: 10.13189/eer.2017.050406

31. Idrissou, Y., Assani, A. S., Toukourou, Y., Worogo, H. S. S., Assogba, B. G. C., Azalou, M., ... \& Alkoiret, I. T. 2019. Systèmes d'élevage pastoraux et changement climatique en Afrique de l'Ouest: Etat des lieux et perspectives.

32. Kabir, I., Dhimal, M., Müller, R., Banik, S., et Haque, U. 2017. L'épidémie de Dhaka chikungunya en 2017. Lancet Infect Dis, 17 (11), 1118.

33. Kahsay et Hansen, (2016). L'impact du changement climatique et de la politique d'adaptation sur la production agricole en Afrique de l'Est, doi : 10.1016 / j.ecolecon.2015.11.016.

34. Katé, S., Dagbenonbakin, G. D., Agbangba, C. E., De Souza, J. F., Kpagbin, G., Azontondé, A., ... \& Sinsin, B. 2014. Perceptions locales de la manifestation des changements climatiques et mesures d'adaptation dans la gestion de la fertilité des sols dans la Commune de Banikoara au Nord-Bénin. Journal of Applied Biosciences, 82(1), 7418-7435.

35. Khanal, U. K., Wilson. C., Wilson, Hoang, V. H., Boon, Lee. 2018. Farmers' Adaptation to Climate Change, Its Determinants and Impacts on Rice Yield in Nepal. Ecological Economics

36. Macauley, H., et Ramadjita, T. 2015. Les cultures céréalières : riz, maïs, millet, sorgho et blé. Africa Rice Center, Benin. 38p.

37. Naswem, A.A., Akpehe, G.A., Awuaga, M.N. 2016. Adaptation Strategies to Climate Change among Rice Farmers in Katsina-Ala Local Government Area of Benue State, Nigeria. IOSR Journal of Agriculture and Veterinary Science (IOSR-JAVS). e-ISSN: 23192380, p-ISSN: 2319-2372. Volume 9, Issue 10 Ver. I (October. 2016), PP 33-37

38. Onyegbula, C. B., et Oladeji, J. O. 2017. Utilization of climate change adaptation strategies among rice farmers in three states of Nigeria. Journal of Agricultural Extension and Rural Development. Vol.9 (10), pp. 223-229, October 2017. DOI: 10.5897/JAERD2017.0895

39. Ouédraogo, M., Dembélé, Y., \& Somé, L. (2010). Perceptions et stratégies d'adaptation aux changements des précipitations : cas des 
paysans du Burkina Faso. Science et changements planétaires/Sécheresse, 21(2), 87-96.

40. Oumarou, Y., Saidou, A. A., Madi, A., Watang Zieba, F. et Fokou Yemata, O. 2017. Perception paysanne des perturbations pluviométriques et stratégies d'adaptation dans les systèmes de culture à sorgho repiqué en zone soudano-sahélienne du Cameroun.

41. Reeves, T. G., Thomas, G. et Ramsay, G. 2016. Produire plus avec moins en pratique : le maïs, le riz, le blé. Guide pour une production céréalière durable.

42. Roco, L. R., Bravo-Ureta, B., Engler, A. et Jara-Rojas, R. 2017. The Impact of Climatic Change Adaptation on Agricultural Productivity in Central Chile: A Stochastic Production Frontier Approach. Sustainability 2017, 9, 1648.

43. Sabugo R. T. 2013. Adaptation strategies by smallholder rice farmers under the influence of climate change: a case of kilombero basin, Tanzania.

44. Shrestha, R. P., Raut, N., Swe L., Maung, M. et Tieng T. 2018. Climate Change Adaptation Strategies in Agriculture: Cases from Southeast Asia. Sustainable Agriculture Research; Vol. 7, No. 3; 2018. doi:10.5539/sar.v7n3p39

45. Skinner M. W. 2002. Adaptation options in agriculture to climate change: a topology. Mitig Adapt Strateg Glob Change. Mitigation and Adaptation Strategies for Global Change, doi: 10.1023/A:1015862228270, pp : 85-114

46. Smit, B., Burton, I., Klein, R. J. T., and Wandel, J. 2000. An anatomy of adaptation to climate change and variability', Climate Change 45, 223-251.

47. Smit et Wandel, 2006 Adaptation, capacité d'adaptation et vulnérabilité Global Environmental Change Volume 16, Issue 3, pages 282-292, doi.org/10.1016/j.gloenvcha.2006.03.008

48. Totin V. S. h., 2005. Tendances hydro climatiques et scénarios de gestion des ressources en eau sur les plateaux du sud-Bénin. Mémoire de DEA EDP/FLASH -UAC.80p.

49. Tse, YK, 1987. A diagnostic test for the multinomial logit model. Journal of Business and Economic Statistics 5 (2), 283-86.

50. Wilbanks et Kates, 1999 Changement global dans les lieux locaux : comment l'échelle joue-t-elle?, Volume 43, Numéro 3 , pp 601628 .

51. Yabi, J. A., Yegbemey, R. N. et Tovignan, S. D. 2017. La gestion des facteurs de production comme une adaptation aux variations climatiques inter saisonnières : Cas de la riziculture au Bénin. 
52. Yegbemey, R. N., Aihounton, G. B. D. et Aloukoutou, A. M. 2019. Providing Smallholder Farmers with Weather-Related Information to Build their Resilience to Climate Variability: A Qualitative Exploration. Tropentag, September 18 - 20, 2019 in Kassel. "Filling gaps and removing traps for sustainable resources development"

53. Zorom Malicki, Barbier Bruno, Mertz Ole, Servat Eric. (2013). Diversification and adaptation strategies to climate variability: A farm typology for the Sahel. Agricultural systems, vol 116. 